\title{
DESIGN AND CHARACTERIZATION OF A COMPACT RADIATION MONITOR FOR SPACE ROCKETS
}

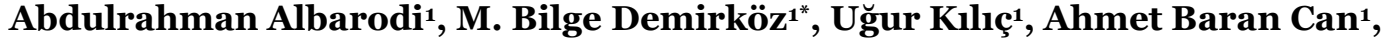 \\ Deniz Orhun Boztemur'1, Egecan Karadöller¹, Aziz Ulvi Çalışkan², \\ Güntekin Kabuli², Levent Balamir Tavacıŏlu²
}

\author{
${ }^{1}$ IVMER (The Research and Application Center for Space and Accelerator Technologies) Middle East Technical \\ University (METU), Ankara, Turkey \\ TÜBİTAK BİLGEM UEKAE YİTAL, Kocaeli, Turkey
}

\begin{abstract}
A compact radiation monitor which incorporates a Geiger-Müller counter and two silicon detectors was designed and tested for radiation measurements on Turkish space rockets. The large area silicon PIN detectors, each with 4 quadrants produced in Turkey by TÜBITTAK BILLGEM UEKAE YİTAL laboratories, vertically aligned inside a thin aluminum shielding, separated by 3 PCBs as degraders, to perform coincidence logic and energy discrimination. Each quadrant is amplified separately to reduce the noise on readout cards designed by METU The Research and Application Center for Space and Accelerator Technologies (IVMER) which generate logical signals per layer, which are then coincided in a 7.8ns time window by an FPGA. The prototype also incorporates a Geiger-Müller tube sensitive to electrons and gammas to compare the counts of particles outside the box measured during test period. The I-V and $C$-V characterization of the PIN diodes, as well as detailed calibration of the readout electronics were performed. The device was tested at the METU-DBL (METU Defocusing Beam Line) proton beam line with 15 and 30 MeV proton beams as well as radioactive alpha and beta sources and shown to be sensitive to different particle species. The dynamic range, which has been demonstrated up to $10^{6}$ particles/second lays the foundation for a robust radiation measurement with more detector and degrader layers for a larger energy range on a satellite over the South Atlantic Anomaly as well as van Allen belts
\end{abstract}

Key words: PIN Diodes, Silicon Detector, Radiation Counter, LEO Radiation Environment, Proton/Heavy Ion Telescope

1. SPACE RADIATION ENVIRONMENT AND COSMIC RAY SHOWERS IN THE ATMOSPHERE

Galactic cosmic rays (GCR) interact with the air and produce particle showers when they enter the Earth's atmosphere $[1,2]$. GCR primaries that are above the geomagnetic cutoff will proceed towards the surface of the Earth while generating secondary particles. Also, these secondaries collide with a higher density of air while they are travelling towards the surface. The resulting particle shower consists of neutrons, electrons, muons, kaons, pions, and neutrinos [4]. Especially, charged muons can reach the Earth's surface without decaying or getting absorbed [3].

Theoretical models and Monte-Carlo simulations of fluxes of secondary particles (muons, electrons and pions) are calculated by CARI7-A using ISO 2004 model for the primary GCR flux at N'42 and E'35 on October 2020 as shown in Figure 1 [5]. The primary flux of protons decreases exponentially as the altitude decreases, while the flux of secondary electrons, positrons, neutrons, muons and pions reach a maximum around an altitude of $20 \mathrm{~km}$ and then decrease rapidly as they approach the Earth's surface. This maximum at $\sim 20 \mathrm{~km}$ is called the Regener-Pfotzer maximum. Measurement of the radiation dose at different altitudes below $35 \mathrm{~km}$ have been performed since the 1950 using balloons and aircrafts frequently.
The accumulated results show a peak in dose rate at altitude of $20 \mathrm{~km}[6,3,7]$.

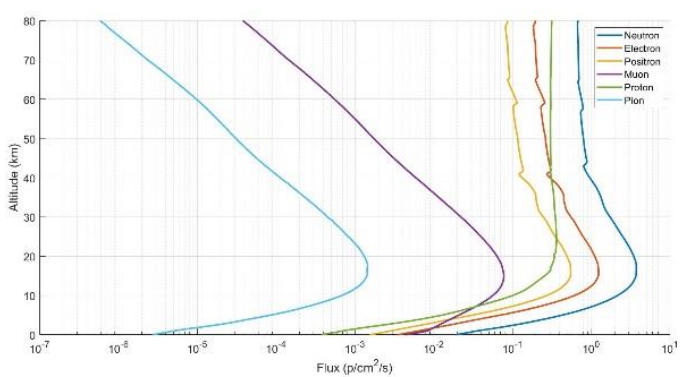

Figure 1. Calculated flux of different particles at different altitude using CARI7-A software with the ISO-2004 model for altitudes above (N'42, E'35) [5].

A summary of the available cosmic radiation data is provided by [3] and is shown in Figure 2. Both dose rate and flux show a clear increase in particle count at around $20 \mathrm{~km}$ as well as in the inner $(1600 \mathrm{~km}$ to 13000 $\mathrm{km}$ ) and outer ( $19000 \mathrm{~km}$ to $40000 \mathrm{~km})$ van Allen belts. Also, the flux in the SAA is two orders of magnitudes higher as measured by the ISS. The specific dose (SD) is a measure of the average energy per particle and changes slightly with altitude as the dominant particle in the GCR spectrum change with altitude. Between 35 and $220 \mathrm{~km}$, there is limited data available because it 
can only be covered by rockets which only spend a short time there [25].
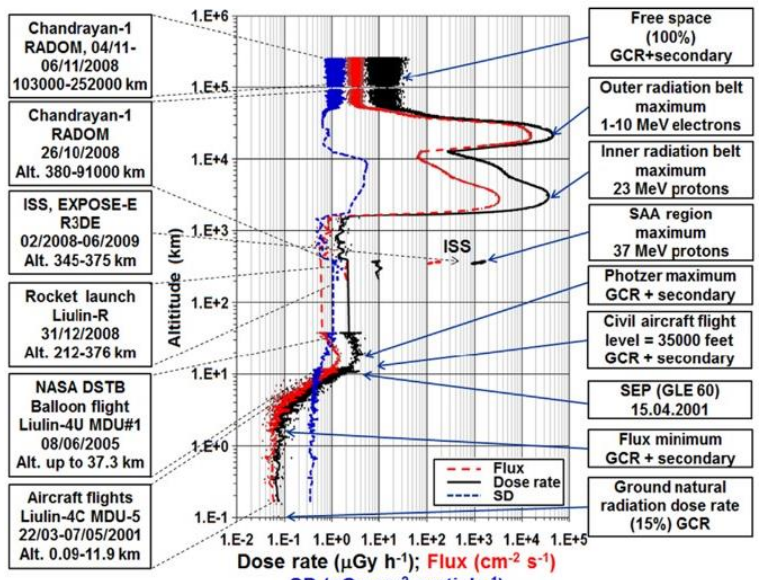

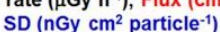

Figure 2. Variations of the absorbed dose rate, flux and specific dose for an altitude range from 0.1 to $250,000 \mathrm{~km}[3]$

\section{PROTOTYPE RADIATION MONITOR (SB.O)}

To measure the dose rate and particle count in high altitudes as well as space, SB.o is designed by METU IVMER. SB.003 and SB.001 were sent to space on $26^{\text {th }}$ and $29^{\text {th }}$ of October 2020 from Sinop, Turkey, in collaboration with ROKETSANTM [9]. This rocket reached an altitude of $136 \mathrm{~km}$. Device employed 2 DDA3 silicon PIN diodes in a vertical alignment to perform coincidence logic and a glass J305 $\beta \gamma$ Geiger-Müller tube to measure particle count outside the box. J305 $\beta \gamma$ tube sensitive to electrons and gammas, has a functional temperature between $-40^{\circ} \mathrm{C}$ and $55^{\circ} \mathrm{C}$, certified for lifetime up to $10^{9}$ particles. SB.o has a mass of $0.5 \mathrm{~kg}$ and a power consumption of $5 \mathrm{~W}$. Mechanical design is shown in Figure 3. This article mainly concerns design, calibration and electrical characteristics of silicon detector system, other details can be found in this referenced thesis [10].

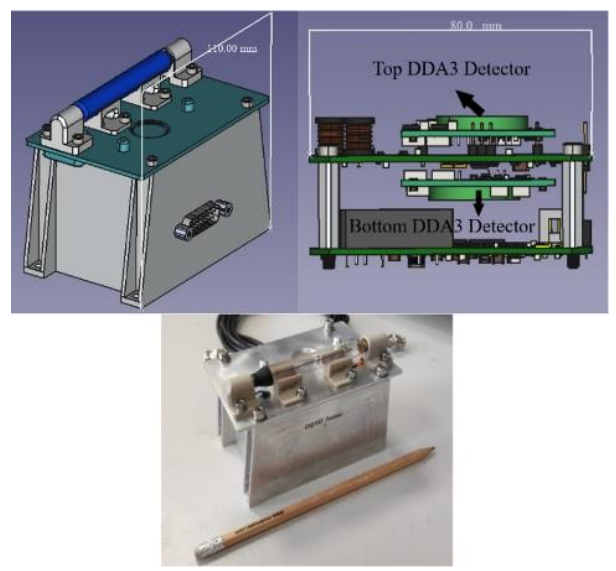

Figure 3. The CAD design of the prototype Radiation Monitor (SB.o) in the upper panel and a picture of the flight module with a pencil for scale in the lower panel.

\subsection{Electronic Characteristics of PIN Diodes}

The properties of the DDA3 and TDA PIN diodes as a particle detector manufactured by TÜBİTAK BILLEM UEKAE YITTAL such as their leakage current and reverse bias relationship (I-V) are measured as well as their reverse bias and capacitance relationship (C-V) characteristics.

The DDA3 detector is circular in shape with a radius of $7 \mathrm{~mm}$ divided into 4 quadrants with a thickness of $0.52 \mathrm{~mm}$. Applying a reverse bias voltage to this detector at room temperature will result in a leakage current as shown in Figure 4. These measurements were taken in the IVMER lab in an ESD-safe dark chamber controlled at $23^{\circ} \mathrm{C}$ since this diode is sensitive to visible light and to temperature changes. The measurements show that the DDA3 detector has a low leakage current $(<3 \mathrm{nA})$ in all its quadrants up to a reverse voltage of $180 \mathrm{~V}$, which is beneficial for low noise particle detection [11].

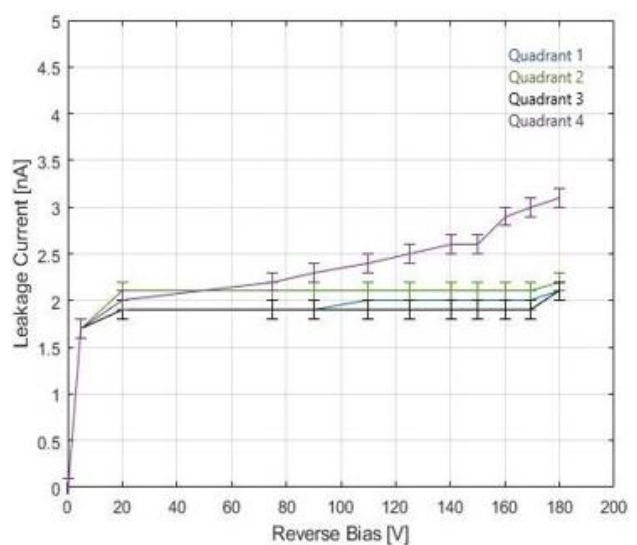

Figure 4. The I-V Characteristics of DDA3 Detector Quadrants.

The TDA detector is another version of DDA3 with same design and processing as well as same area and thickness, but has a single active area, which is also investigated. As the TDA's active area is four times larger, its leakage current expected to be four times larger and the measurement shows that its leakage current is limited to $<20 \mathrm{~A}$.

To achieve a sizable particle signal using the PIN diodes, the reverse bias voltage should be high enough to achieve full depletion, provide high enough electric field to improve the charge collection efficiency and decrease pulse width. Since the breakdown voltage is rated for $200 \mathrm{~V}$ for DDA3 and TDA, $180 \mathrm{~V}$ bias chosen with a $20 \mathrm{~V}$ safety margin.

Leakage current $\left(J_{R}\right)$ of PIN diodes is commonly described in literature as a combination of the diffusion current $\left(J_{\text {Diff }}\right)$ and the generation current $\left(J_{G e n}\right)$ as shown in Equation (1) [12].

$$
\mathrm{J}_{\mathrm{R}}=\mathrm{J}_{\text {Diff }}+\mathrm{J}_{\mathrm{Gen}}=\mathrm{q} \sqrt{\frac{\bar{D}_{\mathrm{p}}}{\tau_{\mathrm{p}}}} \frac{\mathrm{n}_{\mathrm{i}}^{2}}{\mathrm{~N}_{\mathrm{A}}}+\frac{\mathrm{qn}_{\mathrm{i}} \mathrm{W}}{\tau_{\mathrm{e}}}
$$

where $q$ is the charge of the electron, $D_{p}$ is the diffusion coefficient of silicon, $\tau_{p}$ is the minority carrier lifetime, $\tau_{e}$ is the effective lifetime, $n_{i}$ is the intrinsic carrier 
concentration, $\mathrm{N}_{\mathrm{A}}$ is the doping concentration and $\mathrm{W}$ is the depletion width. The depletion width increases with the square root of the reverse bias voltage as described by Equation 2 [12].

$$
\mathrm{W}=\sqrt{\frac{2 \varepsilon_{\mathrm{s}}}{\mathrm{qN}}\left(\mathrm{V}_{\mathrm{bi}}+\mathrm{V}_{\mathrm{R}}\right)}
$$

where $\varepsilon_{\mathrm{S}}$ is permittivity of silicon, $\mathrm{V}_{\mathrm{bi}}$ is the built-in potential of the junction and $V_{R}$ is the reverse bias voltage. Hence, the leakage current of the silicon diode is a function the square root of the reverse bias voltage and can be expressed as in Equation 3 .

$$
\mathrm{J}_{\mathrm{R}}=\mathrm{A}+\mathrm{B} \sqrt{\mathrm{V}_{\mathrm{R}}}
$$

where A and B are fitting parameters representing $J_{\text {Diff }}$ and $\mathrm{J}_{\mathrm{Gen}}$ respectively. The minority carrier lifetime and the effective carrier lifetime are dependent on the temperature and are difficult to estimate theoretically without an injection current measurement. Thus, the effect of temperature on the leakage current must be investigated.

The leakage current dependency of TDA on temperature was investigated inside an oven to heat the detector while the detector attached to a readout PCB. Measurements taken after thermal equilibrium reached as shown in Figure 5. The curves fit to a square root function up to the point of full depletion and then stabilize to a constant value.

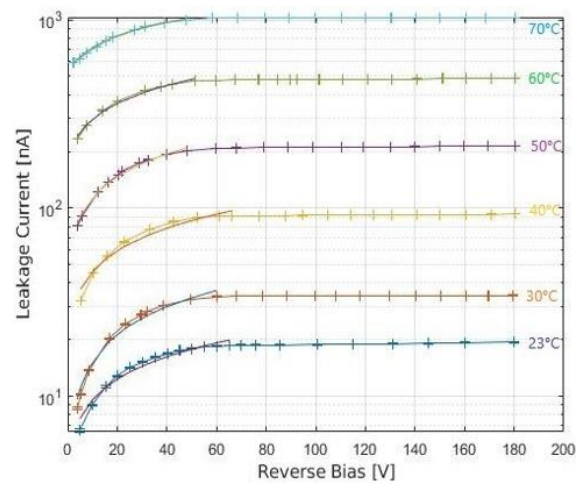

Figure 5. The dependence of the I-V characteristics of TDA detector on temperature where solid lines corresponds to fit functions.

The doping concentration in silicon crystal of the PIN diodes can be determined using these measurements. The curves fit the model in Equation 3 with $\chi^{2}$ values higher than 0.95 for the $\mathrm{A}$ and $\mathrm{B}$ parameters. $A$ defines the leakage current at oV reverse bias and $B$ defines the proportionality of the leakage current to the square root of the reverse bias voltage. The calculations showed that the measurements are conforming with the prediction.

The leakage current of the PIN diode depends on the temperature through Equation 4.

$$
\mathrm{J}_{\mathrm{R}}=\mathrm{C} * \exp \left(\frac{-\mathrm{E}_{\mathrm{a}}}{\mathrm{kT}}\right)
$$

where $C$ is a fitting constant, $\mathrm{E}_{\mathrm{a}}$ is activation energy, $\mathrm{k}$ is Boltzmann's constant and $T$ is the temperature in kelvins. Hence, a plot of the logarithm of the leakage current versus $1 / \mathrm{kT}$ should be linear with a slope of $-\mathrm{E}_{\mathrm{a}}$. Plot for different reverse bias voltage values are presented in Figure 6 and values calculated for $E_{a}$ are shown in Table 1. According to these results the full depletion is reached at $40 \pm 5 \mathrm{~V}$ of reverse bias since the chance in $E_{a}$ switches from exponential to linear with very low slope.

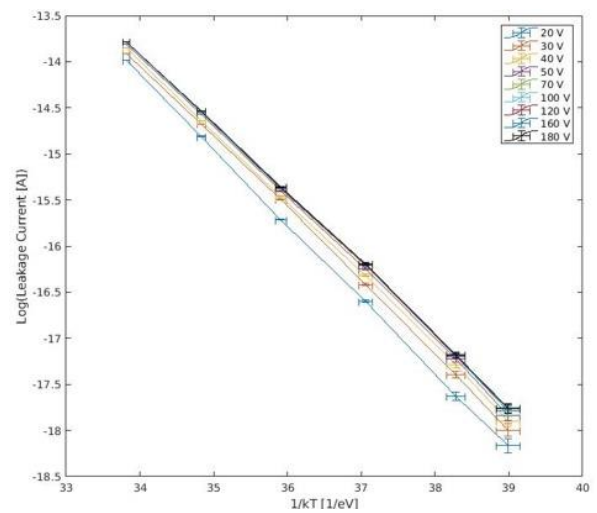

Figure 6. Linear fits to the logarithm of leakage current against $1 / k T$ for activation energy calculation of the TDA detector.

Table 1. Activation Energies $\left(\boldsymbol{E}_{\boldsymbol{a}}\right)$ for different Reverse Bias Voltages $\left(\boldsymbol{V}_{\boldsymbol{R}}\right)$ as Calculated by Fitting of the Data in Figure 6 to Equation 4.

\begin{tabular}{|c|c|c|c|}
\hline$V_{R}[\mathrm{~V}]$ & $E_{a}[\mathrm{eV}]$ & $V_{R}[\mathrm{~V}]$ & $E_{a}[\mathrm{eV}]$ \\
\hline $20.0 \pm 0.1$ & $0.81 \pm 0.01$ & $100.0 \pm 0.1$ & $0.77 \pm 0.01$ \\
\hline $30.0 \pm 0.1$ & $0.79 \pm 0.01$ & $120.0 \pm 0.1$ & $0.77 \pm 0.01$ \\
\hline $40.0 \pm 0.1$ & $0.78 \pm 0.01$ & $160.0 \pm 0.1$ & $0.77 \pm 0.01$ \\
\hline $50.0 \pm 0.1$ & $0.78 \pm 0.01$ & $180.0 \pm 0.1$ & $0.77 \pm 0.01$ \\
\hline $70.0 \pm 0.1$ & $0.77 \pm 0.01$ & & \\
\hline
\end{tabular}

The capacitance of detector versus reverse bias voltage changes can show the full depletion width and with the I-V curve provide information about the doping of the PIN diodes. The $\mathrm{C}-\mathrm{V}$ curve was measured by a semiconductor characterization device in BILGEM UEKAE YITAL. Although, error bars are not provided by the device for error propagation analysis, Figure 7 shows that full depletion occurs for both detectors at $40 \pm 5 \mathrm{~V}$.

The capacitance $C$ of the detector is related to the reverse bias voltage with Equation 5 [13]

$$
\mathrm{C}=\mathrm{qAN}_{\mathrm{A}}(\mathrm{W}) \frac{\mathrm{dW}}{\mathrm{dV}}
$$

where $N_{A}(W)$ is the doping concentration as a function of the depletion width. Hence, $C$ is directly dependent on the derivative of the depletion width with respect to the reverse bias voltage $\frac{d W}{d V_{R}}$. The depletion width $W$ dependence on the capacitance is described by Equation 6.

$$
\mathrm{W}=\frac{\mathrm{K}_{\mathrm{s}} \varepsilon_{0} \mathrm{~A}}{\mathrm{C}}
$$

where $K_{s}$ is the semiconductor dielectric constant, $\varepsilon_{0}$ is permittivity of free space. Thus, the doping concentration can be written as shown in Equation 7 .

$$
\mathrm{N}_{\mathrm{A}}(\mathrm{W})=\frac{2}{\mathrm{qK}_{\mathrm{S}} \varepsilon_{0} \mathrm{~A}^{2} * \mathrm{~d}\left(1 / \mathrm{C}^{2}\right) / \mathrm{dV}}
$$


Here, the doping concentration depends on the slope of the $1 / \mathrm{C}^{2}$ versus $\mathrm{V}$ curve. The calculations showed that the doping concentration of $2-8 * 10^{12}$ ions $/ \mathrm{cm}^{3}$ agree with the specifications provided by the YITAL team.
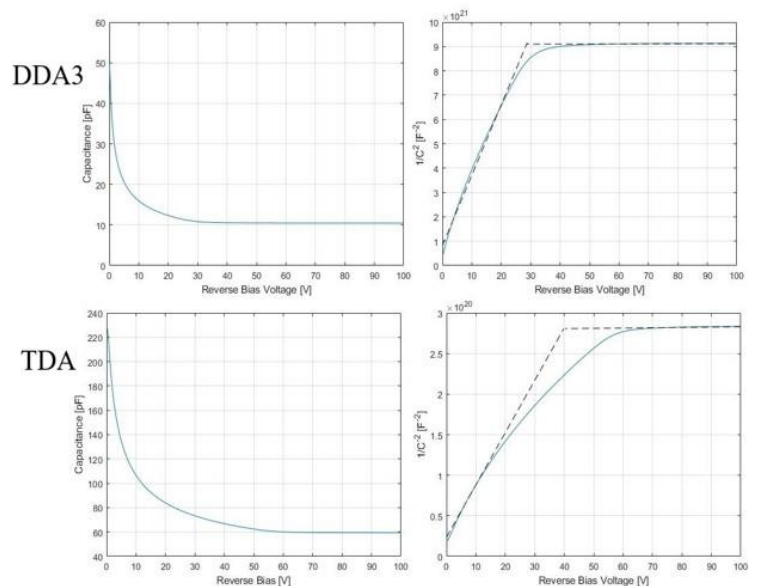

Figure 7. C-V (upper left) and $1 / \mathrm{C}^{2}-\mathrm{V}$ (upper right) plots for a single quadrant in a DDA3 detector and $\mathrm{C}-\mathrm{V}$ (lower left) and $1 / \mathrm{C}^{2}-\mathrm{V}$ (lower right) plots for the TDA detector.

The expression in Equation 7 is only valid until the full depletion point [15]. Variation of the doping concentration from the model depends on many factors such as the internal geometry of the PIN diode layers and the quality of the silicon crystal manufacturing process. The doping concentration of the DDA3 and TDA detectors does not change much inside the silicon crystals for the entirety of its depth since they are grown in the same homogenous and mostly pure environment. An impurity concentration in the range of $10^{11}-10^{12}$ $\mathrm{cm}^{-3}$ is considered to be very pure.

\subsection{Readout Electronics and Calibration of DDA3 as a Particle Detector}

The signal from each quadrant of DDA3 was amplified separately and joined together by using a OR logic gate just before sending signal to IGLOO2 FPGA to count particles and coincidences between upper and lower detectors. The readout circuit has two amplifying and one discrimination stage which employs commercial components. The first trans-impedance amplifier transforms the current signal into a voltage pulse and amplifies it, while the second amplifier has a gain of 1.3 and serves mostly as an inverter [14]. The amplified signal is then fed into a discriminator that has a single noise eliminating threshold. The discriminator also employs an external hysteresis positive feedback loop that in addition to its own provides an additional $41 \mathrm{mV}$ to its internal $9 \mathrm{mV}$ hysteresis value. This helps the discriminator to work sensitively against noise levels close to the reference voltage.

SB.o is expected to be exposed to only cosmic rays penetrating into the upper atmosphere and their secondary's on a sounding rocket. The minimum ionizing particles (MIP) in those high altitudes are the sensitivity target for this detector design, since the energy deposition of the MIP's is lower limit of all other 128 particles for same species. This means that if a radiation monitor can measure MIP's of certain species, it is also able to measure all primaries of that species regardless of kinetic energy.

To find deposited energy vs voltage calibration constant of SB:O's analog signal, alpha particles from Am-241 used. The voltage pulse histograms were taken for one minute intervals using the analog signal after inverting amplifier. Each histogram represents a different deposited energy because measurements were performed in the air medium where alphas lose energy dramatically, therefore each different distance setting between detector and source represents a different deposited energy on the detector by alphas. The results are shown in Figure 8 and the number of counts fall as expected with increasing since the intensity of the radiation depends on $r^{2}$.

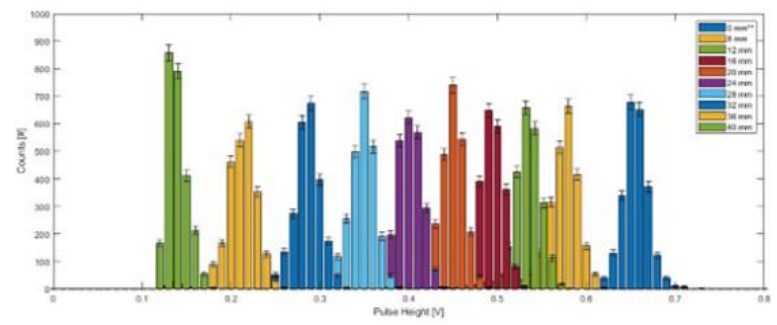

Figure 8. Measured pulse height of Am-241 alpha particles from different distances. The measurement at omm indicates measurement done in vacuum $\left(5 * \mathbf{1 0}^{-2}\right.$ Torr $)$.

The test also simulated using Geant4 taking in to account the air density in Ankara, Turkey at the time as $\rho_{\text {air }}=1.08 \mathrm{~kg} / \mathrm{m}^{3}[16,17]$. The maximum range of an alpha particle from Am-241 in silicon is calculated as $28 \mu \mathrm{m}$ using SRIM [18]. The Geant4 simulation result for energy deposition were compared with the measurements of the pulse heights at different distances as shown in Figure 9. This plot shows the linear correlation with ratio $116.2 \mathrm{mV} / \mathrm{MeV}$ between the energy deposited in the detector and the measured pulse height to be used in determining the particle energy for a given pulse height.

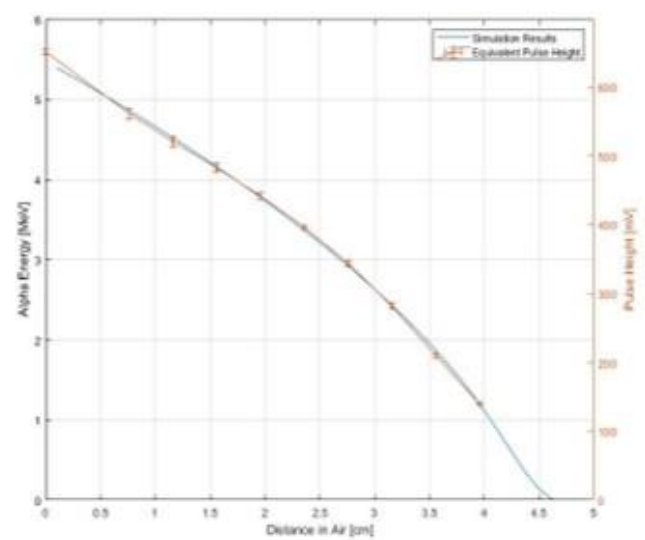

Figure 9. The similar behavior of Geant4 simulation results of alpha energy deposited in the detector and Am-241 alpha particle pulse heights at different distances between the source and the detector demonstrated a linear correlation with a ratio $116.2 \mathrm{mV} / \mathrm{MeV}$. 
The energy deposition of the common cosmic ray MIPs was also simulated using Geant4 for different particles and the equivalent pulse heights were calculated as presented in Table 2.

Table 2. Energy depositions of common cosmic ray MIP's in the detector from Geant 4 and the calculated corresponding equivalent pulse height from the calibration.

\begin{tabular}{|c|c|c|c|}
\hline Particle & Source & $\begin{array}{c}\text { Mean Energy } \\
\text { Deposition } \\
{[\mathrm{MeV}]}\end{array}$ & $\begin{array}{c}\text { Equivalent } \\
\text { Pulse Height } \\
{[\mathrm{mV}]}\end{array}$ \\
\hline Alpha & $\begin{array}{c}\text { Am-241 } \\
(1.72 \mathrm{~mm})\end{array}$ & $3.92 \pm 0.01$ & $457.3 \pm 1.2$ \\
\hline Alpha & MIP & $1.40 \pm 0.04$ & $161.2 \pm 4.7$ \\
\hline Proton & MIP & $0.43 \pm 0.05$ & $50.1 \pm 5.8$ \\
\hline Muon & MIP & $0.25 \pm 0.03$ & $29.2 \pm 3.5$ \\
\hline
\end{tabular}

The noise in the detector readout must be lower than the equivalent pulse height for the particles in question for a reliable readout. Therefore, noise in the readout was measured by changing the reference voltage at the discriminator and measuring the number of counts per minute at the OR logic gate output. A threshold scan was performed using an Am-241 source and the measurement showed that between $74 \mathrm{mV}$ and $500 \mathrm{mV}$ reference voltages, number of counts did not change. Also, without any radiation source, the noise count rate was less than 1 count/minute for reference voltages higher than $74 \mathrm{mV}$. Hence, the threshold was set at $74 \mathrm{mV}$. The lower energy cut off for protons is $13 \mathrm{MeV}$ due to $1 \mathrm{~mm}$ thick shielding above the detector and the higher cut off energy is $35 \mathrm{oMeV}$ for proton due to the $74 \mathrm{mV}$ threshold.

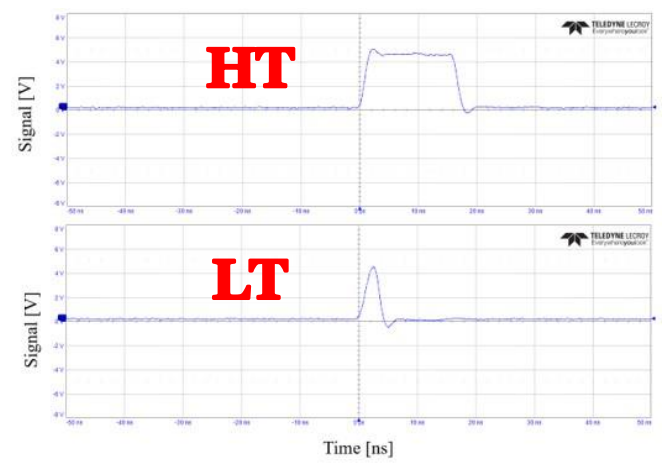

Figure 10. Comparison between low energy or noise hit (below) and a high energy or stopping particle (above) signal shapes at the discriminator output.

SB.o employed an IGLOO2 FPGA which was operated with a $128 \mathrm{MHz}$ (7.8ns period) clock [19]. For silicone PIN diodes, using a single voltage threshold, particle counting algorithm that classifies high (HT) and therefore long and low (LT) and therefore short signals was implemented to distinguish low and high $d E / d x$ of particles in the detectors. HT corresponded to 3 clock cycles and LT corresponded to 1 clock cycle analog signals determining the energy deposition of the particles. An alpha particle which deposited $\sim 3.9 \mathrm{MeV}$ caused 3 cycle analog signal (HT) whereas a proton which deposited $\sim 0.64 \mathrm{MeV}$ resulted in 1 cycle analog signal (LT) as seen in the Figure 10. For the GeigerMuller Tube straightforward counting was applied considering the deadtime of the tube. FPGA readout communication was done using UART, sending 64 byte/ 1 second packages including coincidence, sum of counts, per second counts and CRC data.

\subsection{Performance Tests}

To test SB's sensitivity to different particles, the Geiger counter and DDA3 detectors were exposed to different radiation sources. SB.001 and SB.003 are flight modules while SB.002 was the module used for the environmental and flight-readiness tests. The tests were performed in the IVMER lab and the sources were held $1.0 \mathrm{~cm}$ away from the Geiger counter and $2.0 \mathrm{~cm}$ away from the DDA3 detectors. The sources used are listed below:

- $37 \mathrm{kBq}$ Cs137 electron source

- $406 \mathrm{MBq}$ Co57 gamma source

- $74 \mathrm{kBq}$ Am241 alpha source (for Geiger counter)

- 3.7 kBq Am241 alpha source (for DDA3)

A DDA3 detector and its readout electronics were tested in the proton beam test facility of METU DBL as well $[20,21,22] .15 \mathrm{MeV}$ and $30 \mathrm{MeV}$ primary proton beams with fluxes $\sim 10^{8} \mathrm{p} / \mathrm{cm}^{2} / \mathrm{s}$ exit the METU-DBL through a $50 \mu \mathrm{m}$ titanium window and travel about $5 \mathrm{~cm}$ before they hit the target area. The radiation testing of components was performed at METU-DBL facility and no malfunctions were observed. The detector readout card was housed in a shielded enclosure with aluminum plates and polyethylene blocks to shield the card against protons and secondary neutrons. The shielded enclosure had a straight aperture to fit the DDA3 detector such that the protons can pass to the sensitive area, and the aperture was sealed with $100 \mu \mathrm{m}$ aluminum foil to block light from reaching the DDA3 detector. The signal was readout using a RedpitayaTM 125-14 card at a sampling rate of $125 \mathrm{MHz}$ [23] and was sent via SCPI server to a computer running a script for analysis. Also, the energy deposition of $15 \mathrm{MeV}$ and 30 $\mathrm{MeV}$ protons in the DDA3 detectors were calculated using detailed Geant4 simulations which includes energy loss in the titanium and aluminum windows as well as air. The ratio of simulated energy and measured different pulse widths, but only the pulse widths above the threshold for both 15 and $30 \mathrm{MeV}$ protons are slightly different but within error bars which shows the correlation between them. Results are summarized in Table 3 .

Table 3. Summary and comparison of the energy deposition values obtained via Geant4 and the measured signal widths, but only the pulse widths above the threshold in METU-DBL

\begin{tabular}{|c|c|c|c|}
\hline & $15 \mathrm{MeV}$ & $30 \mathrm{MeV}$ & Ratio \\
\hline $\begin{array}{c}\text { Mean } \\
\text { Energy } \\
\text { Deposition } \\
{[\mathrm{MeV}]}\end{array}$ & $3.7 \pm 0.4$ & $1.9 \pm 0.1$ & $1.95_{-0.30}^{+0.33}$ \\
\hline $\begin{array}{c}\text { Mean } \\
\text { Signal } \\
\begin{array}{c}\text { Width } \\
\text { [Sample] }\end{array}\end{array}$ & $10.0 \pm 0.2$ & $4.2 \pm 0.1$ & $2.38_{-0.10}^{+0.11}$ \\
\hline
\end{tabular}


The signal width above the threshold does not correspond linearly to the deposited energy. Rather, it depends mostly on the feedback capacitance in the trans-impedance amplification stage and the RC constant of the readout electronics which plays a big role in determining the shape of the peak based on the current pulse through a non-linear relationship [24].

Another DDA3 and its readout electronics from the SB.002 box remained connected to the FPGA inside the $\mathrm{SB}$ enclosure and was positioned away from the main irradiation area of the beam such that it is exposed to secondary particle irradiation. The upper aluminum shielding of the SB enclosure was removed and replaced by $100 \mu m$ aluminum foil to let as many secondaries through as possible without exposing the DDA3 detector to light. The results show that this system has a wide dynamic range as a radiation monitor and can easily measure particle rates at $10^{6}$ particles/s, which shows it can function in the South Atlantic Anomaly (SAA) and the poles.

\section{CONCLUSION}

In this article PIN diode characteristics, readout electronics and FPGA logic algorithms of "SB.o" were presented as a compact radiation monitor. SB.o is designed to measure the dose rate and particle count in the atmosphere at high altitudes as well as space. The performance tests in METU IVMER laboratory and METU DBL proton beamline were proved that SB.o can measure particles up to $10^{6}$ particles/s and has a wide dynamic range. In the ROKETSANTM flights, SB.o was able to detect Regener-Pfotzer maximum at $\sim 20 \mathrm{~km}$ with a coincidence data conforming the telescope logic of the vertically aligned PIN diodes. The results of the sounding rocket flights can be found in this referenced conference [8].

Acknowledgements: The authors would like to thank TENMAK PAF for help with performing radiation tests. This research was supported by the Presidency of Strategy and Budget project number 2015K12119o. This work was in part funded by the Turkish Academy of Sciences (TÜBA).

\section{REFERENCES}

1. T. Sato, "Analytical model for estimating terrestrial cosmic ray fluxes nearly anytime and anywhere in the world: extension of PARMA/EXPACS," PLoS One, vol. 10, no. 12, article no. e0144679, Dec. 2015. https://doi.org/10.1371/journal.pone.0144679

2. R. Engel, D. Heck, T. Pierog, "Extensive air showers and hadronic interactions at high energy," Annu. Rev. Nucl. Part. Sci., vol. 61, pp. 467-489, Nov. 2011. https://doi.org/10.1146/annurev.nucl.o12809.104544

3. T. P. Dachev, "Profile of the ionizing radiation exposure between the Earth surface and free space," J. Atmos. Sol.-Terr. Phys., vol. 102, pp. 148-156, Sep. 2013. https://doi.org/10.1016/j.jastp.2013.05.015

4. M. Barrantes et al., "Atmospheric corrections of the cosmic ray fluxes detected by the Solar Neutron Telescope at the Summit of the Sierra Negra Volcano in Mexico," Geofis. Int., vol. 57, no. 4, pp. 253-275, Oct. 2018. https://doi.org/10.22201/igeof.00167169p.2018.57.4.21 $\underline{05}$

5. K. Copeland, "CARI-7A: development and validation," Radiat. Prot. Dosim., vol. 175, no. 4, pp. 419-431, Aug. 2017 https://doi.org/10.1093/rpd/ncw369

6. Y. I. Stozhkov, N. S. Svirzhevsky, V. S. Makhmutov, "Cosmic ray measurement in the atmosphere," in Proc. Workshop on Ion-Aerosol-Cloud Interact (IACI), Geneva, Switzerland, 2001, pp. 41-62. https://doi.org/10.5170/CERN-2001-O07

7. R. G. Harrison, K. A. Nicol, K. L. Aplin, "Vertical profile measurements of lower troposphere ionization," J. Atmos. Sol.-Terr. Phys., vol. 119, pp. 203-210, Nov. 2014. https://doi.org/10.1016/j.jastp.2014.08.006

8. M. B. Demirköz et al., "Design of a space radiation monitor for a sounding rocket and results from the first Turkish sounding rocket flight," presented at the Rad. Effects on Components and Systems (RADECS), Vienna, Austria, Sep. 2021.

9. "Türk roketi ilk kez sıvı yakıt ile uzayda," ROKETSAN Haber, Kas. 13, 2020.

("Turkish rocket in space for the first time with liquid fuel," ROKETSAN News, Nov. 13, 2020.)

Retrieved from:

https://www.roketsan.com.tr/tr/medya/haberler/turkroketi-ilk-kez-sivi-yakitla-uzayda

Retrieved on: Nov. 13, 2020

10. A. Albarodi, "Design of a space radiation monitor for a spacecraft in LEO and results from a prototype on the first Turkish sounding rocket”, M.Sc. dissertation, Middle East Technical University, Dept. of Physics, Ankara, Turkey, 2021.

Retrieved from:

http://etd.lib.metu.edu.tr/upload/12626153/index.pdf Retrieved on: May 25, 2021

11. S. Srivastava, R. Henry, A. Topka R., "Characterization of PIN diode silicon radiation detector," Int. J. Intell. Electr. Syst., vol. 1, no. 1, pp. 47-51, 2007.

https://doi.org/10.18000/ijies.30009

12. J. M. Park et al., "Consideration of the Leakage-Current and the Radiation-Response characteristics of silicon PIN detectors with different N-Type Substrates and Their Application to a Personal $\gamma$-ray dosimeter," $J$. Korean Phys. Soc., vol. 51, no. 1, pp. 10-17, 2007. https://doi.org/10.3938/jkps.51.10

13. D. K. Schroder, "Carrier and Doping Density," in Semiconductor Material and Device Characterization, $3^{\text {rd }}$ ed., New Jersey, USA, J. Wiley and Sons, 2006, ch. 2, sec. 2, pp. 61-78. https://doi.org/10.1002/0471749095

14. T. L. Floyd, D. Buchla, "Basic Op-amp Circuits" in Fundamentals of Analog Circuits, $2^{\text {nd }}$ ed., USA, Prentice Hall, Pearson, 2002, ch. 8, ch. 1-4, pp. 418-445.

15. R. Gaillard, "Single Event Effects: Mechanisms and Classification," in Soft Errors in Modern Electronic Systems, $1^{\text {st }}$ ed., Boston, MA, USA, Springer, 2011, ch. 2, pp. 27-54. https://doi.org/10.1007/978-1-4419-6993-4 2

16. Geant4 Collaboration, Geant4 User's Guide for Application Developers, Geant4 version 10.3, CERN, Geneva, Switzerland, 2016.

Retrieved from:

https://geant4-

userdoc.web.cern.ch/UsersGuides/ForApplicationDevel oper/BackupVersions/V10.3/html/index.html Retrieved on: Jul. 15, 2020

17. M. Pinto, P. Goncalves, "GUIMesh: A tool to import STEP geometries into Geant4 via GDML," Comp. Phys. Commun., vol. 239, pp. 150-156, 2019. https://doi.org/10.1016/i.cpc.2019.01.024 
M. B. Demirköz et al., A compact radiation monitor for space rockets ..., RAD Conf. Proc., vol. 5, 2021, 125-131

18. J. F. Ziegler, M. D. Ziegler, J. P. Biersack, "SRIM - The stopping and range of ions in matter," Nucl. Instrum. Methods Phys. Res. Sec. B: Beam Interact. Mater. At., vol. 268, no. 11-12, pp. 1818-1823, Jun. 2010. https://doi.org/10.1016/j.nimb.2010.02.091

19. Microsemi, DSO128: IGLOO2 and SmartFusion2 Datasheet, $12^{\text {th }}$ ed., Microchip, California, USA, 2008 Retrieved from:

https://www.microsemi.com/documentportal/doc download/132042-igloo2-fpga-datasheet Retrieved on: Aug. 15, 2020

20. A. Gencer, M. B. Demirkoz, I. Efthymiopoulos, M. Yiğitoğlu, "Defocusing beam line design for an irradiation facility at the TAEA SANAEM Proton Accelerator Facility," Nucl. Instrum. Methods Phys. Res. Sec. A: Accel. Spectrom. Detect. Assoc. Equip., vol. 824, pp. 202-203, Jul. 2016 https://doi.org/10.1016/j.nima.2015.11.018

21. M. B. Demirkoz, S. Niğdelioğlu, M. Yiğitoğlu, S. Aydın, I. Efthymiopoulos, "METU defocusing beam line project for the first SEE tests in Turkey and the results from the METU-DBL preliminary setup," Nucl. Instrum. Methods
Phys. Res. Sec. A: Accel. Spectrom. Detect. Assoc. Equip., vol. 936, pp. 54-56, Aug. 2018.

https://doi.org/10.1016/j.nima.2018.11.075

22. M. B. Demirkoz et al., "METU-Defocusing beamline: A 15-30 MeV proton irradiation facility and beam measurement system," EPJ Web Conf., vol. 225, article no. 01008, Jan. 2020.

https://doi.org/10.1051/epjconf/202022501008

23. Red Pitaya Documentation, Redpitaya, Slovenia, 2020. Retrieved from: https://redpitaya.readthedocs.io/ Retrieved on: May 25, 2020

24. Z. Bielecki, "Readout electronics for optical detectors", Opto-Electron. Rev., vol. 12, no. 1, pp. 129-137, 2004. Retrieved from:

https://www.researchgate.net/publication/228798113 Readout electronics for optical detectors Retrieved on: Dec. 20,2020

25. M. Wijtvliet et al., "PR3: A system for radiointerferometry and radiation measurement on sounding rockets," Microprocessors and Microsystems, vol. 77, article no. 103163, Sep. 2020.

https://doi.org/10.1016/j.micpro.2020.103163 